\title{
Ciberhinchas, ciberaficionados, ciber-tifosi: internet, nuevo espacio de expresión futbolera
}

Claudia BENASSINI

\section{INTRODUCCIÓN}

A mediados del siglo pasado los ingleses se encargaron de crear y reglamentar el football, que encuentra en el calcio (fútbol en italiano) su precursor. Según Fernando Marcos, el momento culminante se dio en 1948 en Cambridge, donde se llevó a cabo una reunión de representantes de varias universidades de Inglaterra para pactar un código que les permitiera competir entre sí.

Las Reglas de Cambridge contenian 14 puntos que, con algunas modificaciones, rigen aún al deporte más popular del siglo XX. Los británicos no tardaron en llevar su juego a todas las naciones del mundo. Estudiantes, marinos, ejecutivos de ultramar y técnicos especialistas en los nuevos procesos industriales fueron los portadores de este raro contagio; en breve lo propagaron por la Europa continental (Suiza, Bélgica, España, Asia y América) (Marcos, en Bañuelos Rentería, 1998:8).

En México, el deporte hizo su aparición un par de décadas más tarde, como producto de la oleada británica que caracterizó los primeros años del régimen de Porfirio Díaz.

En las minas de Real del Monte y Pachuca, en las fábricas y cafetos de Veracruz, en los campos del Casino Inglés y el British Club de la capital de la República comenzaron a rodar, cuando apenas despuntaba el siglo que concluye, los primeros balones de cuero. Fueron los 
ingleses, que apenas cuatro décadas atrás habían fundado el primer equipo de soccer en el mundo (el Sheffield Club) los primeros que practicaron este deporte entre nosotros (Marcos, en Bañuelos Rentería, 1998:8-9)

Y fundaron el Pachuca Athletic Club. Sin embargo, todo indica que, en su desarrollo y consolidación, México recibió el empujón más importante de España, cuyo club fue precursor y semillero de lo que décadas más tarde sería el deporte más importante de nuestro país. En pocos años surgieron diversos equipos, primero en la Ciudad de México y más adelante en el interior como Guadalajara y Veracruz. El auge del fútbol propició que rápidamente comenzara a jugarse no sólo por profesionales sino también por aficionados que, principalmente ubicados en las colonias populares de la Ciudad de México comenzaron a practicarlo en vecindades, terrenos baldíos o en las fábricas en las que prestaban sus servicios en calidad de obreros. Esta nueva modalidad rápidamente permitió que los aficionados con posibilidades pasaran a formar parte de los equipos profesionales, a la vez que se engrosaban las filas de hinchas que se mostraban adeptos a tal o cual equipo.

Asimismo, de manera creciente los medios de comunicación comenzaron a tratar el tema del fútbol. Según Javier Bañuelos Rentería (1998:60), desde principios de siglo, los principales periódicos de entonces -como El Imparcial y El Mundo Ilustrado- inauguraron sendas columnas sobre el tema. El mismo autor señala que de 1918 en adelante aparecieron revistas especializadas: Toros y Deportes (1925) y $\mathrm{La}$ Afición fueron las más importantes por su papel en la consolidación del fútbol. Por su parte y una vez consolidada como industria, la radio inicia las primeras transmisiones desde los estadios y a mediados de la década de 1950 sucede lo mismo con la televisión. En ambos casos la finalidad era clara: llegar a los hogares del creciente número de seguidores que por diversas razones no podian presenciar en vivo el de-

1. Una de las películas más representativas del género es posiblemente Los hijos de don Venancio, pues evoca el momento culminante de la influencia española en el fútbol mexicano. sempeño de su equipo o deporte favorito. Por su parte, el cine se ha ocupado del tema en diversas ocasiones, particularmente exaltando la figura de los ídolos futboleros del momento ${ }^{1}$.

Ciertamente, los medios de comunicación han jugado un papel muy importante en la difusión y consolidación del fútbol, tanto mexicano como de otros países, en los que el deporte se practica con la misma popularidad. Este papel va más allá de lo puramente informativo, en la medida en que se constituye en un importante espacio para que la afición 
encuentre en ellos el punto de vista que comparten o del que discrepan. Sin embargo, salvo excepciones, los medios se han constituido en espacios de expresión para los hinchas, quienes han tenido que conformarse con las modalidades tradicionales del correo a medios impresos, la llamada telefónica a los programas de radio y televisión o el saludo a los amigos gracias a la cámara indiscreta que los capta en el estadio. Cabe señalar que hay excepciones que han permitido que los hinchas expresen sus opiniones futbolísticas a través de medios informales ${ }^{2}$.

Por otra parte, el advenimiento de la era digital, con el consecuente auge y consolidación de internet como medio de comunicación, ha propiciado que entre otros usos proliferen el informativo y el entretenimiento. Una revisión a las principales páginas sobre fútbol muestra que es creciente el número de ciber-tifosi que por diversos motivos cuenta con una página sobre el tema, la misma que actualizan y retroalimentan en función de su tiempo libre, sus intereses y sus necesidades. Este trabajo tiene como finalidad presentar los resultados de una encuesta aplicada a 91 aficionados al fútbol que cuentan con páginas de internet, ubicados en diversas partes del mundo, principalmente Europa y América Latina. Estos resultados permitirán ver las nuevas posibilidades abiertas a la afición por la red de redes, las mismas que posiblemente rebasan las oportunidades ofrecidas por los medios tradicionales. Previamente se presenta el contexto de la sociedad digital y de los nuevos medios de comunicación. En mayor o menor medida, ambos elementos son corresponsables de esta nueva forma de expresión que rápidamente se extiende por todo el mundo.

\section{LA SOCIEDAD DIGITAL 3}

Una de las consecuencias más importantes de la globalización ${ }^{4}$, al menos desde la perspectiva de las comunicaciones, es el paso de la sociedad de masas a la sociedad digital. Una de las características más importantes de la primera fue el concepto de producción en serie,

con economías basadas en una producción realizada con métodos uniformes y repetitivos, en cualquier espacio y tiempo dado (...). En la era de la información, los medios masivos se volvieron, a un mismo tiempo, más grandes y más pequeños. Nuevas formas de emisión (...) llegaron a audiencias cada vez mayores, ampliando el concepto y la forma de la transmisión. Las revistas espe-
2. Por ejemplo, existen casos documentados sobre cómo algunos hinchas se unen para estructurar su propio periódico en el que primordialmente difunden información sobre su equipo favorito $\mathrm{y}$, de manera creciente, sobre el resto de la jornada. En España, particularmente en Barcelona, se registraron experiencias durante la década pasada en que los hinchas podian producir programas de televisión con periodicidad y duración variables.

\section{Parte de la información contenida en este apartado fue trabajada a partir de Benassini, 1997.}

4. En este contexto, estamos de acuerdo con la definición de globalización proporcionada por Anthony Giddens: “... la intensificación de las relaciones sociales en todo el mundo por lo que se enlazan lugares lejanos, de tal manera que los acontecimientos locales están configurados por acontecimientos que 
ocurren a muchos kilómetros de distancia o viceversa. Éste es un proceso dialéctico puesto que esos acontecimientos locales pueden moverse en dirección inversa a las distintas relaciones que les dieron forma (Giddens, 1994:68). cializadas, los videocasetes y los servicios de cable fueron ejemplos de la sectorización, que se dirigieron a grupos demográficos específicos y reducidos. Los medios crecieron y, al mismo tiempo, se achicaron (Negroponte, 1996: 181-182).

\section{Características}

En la sociedad digitalizada todo se hace a pedido y la información está personalizada al máximo.

\begin{abstract}
Una suposición muy difundida es que la individualización es la extrapolación de la sectorización: se va de un grupo grande a uno más pequeño y de ahí a otro más pequeño aún hasta que, por último, el destinatario es un solo individuo (...). Esta línea de razonamiento deja de lado, por completo, la diferencia fundamental entre sectorización y digitalización (Negroponte, 1996:181-182).
\end{abstract}

Es decir, la digitalización incluye datos sobre la persona que determinan el tipo de servicios a recibir en comunidades cada vez más reducidas.

Negroponte coincide con otros autores (Mattelart, 1996) en que la sociedad digitalizada se caracteriza por una eliminación de las fronteras geográficas.

De la misma manera que el hipertexto anula las limitaciones de la página impresa, la era de la post-información anulará las limitaciones geográficas. La vida digitalizada nos hará cada vez más independientes del hecho de tener que estar en un lugar específico, en un momento determinado. Asimismo (el concepto de dirección) cobrará un nuevo significado, dado que será posible vivir y trabajar en una o varias locaciones geográficas distintas" (Negroponte, 1996:183-184).

Es evidente el papel que juegan los medios de comunicación en este proceso, sobre todo en términos de simultaneidad en la propagación de ideas e información, aunque en la nueva sociedad también han sufrido modificaciones que se revisarán en el siguiente apartado.

\section{Los nuevos medios de comunicación}

Posiblemente el cambio más importante que se ha registrado en las últimas décadas es el paso del sistema analógico al digital. 
Las variaciones similares de magnitudes diversas (por ejemplo, corriente eléctrica y presión ejercitada por la emisión oral sobre el diafragma del teléfono) han sido sustituidas por sus cuantificaciones numéricas, permitiendo así, por una parte, la transmisión de muchas más señales simultáneamente por el mismo canal y, por la otra, la posibilidad de transportar, por el mismo canal, señales no homogéneas entre sí, pero convertidas en similares y reciprocamente compatibles, precisamente por su reducción a entidades numéricas (Betettini y Colombo, 1995:15-16).

Esta característica repercutirá directamente en las modalidades de funcionamiento de los nuevos medios.

A esta nueva posibilidad cabe añadir otra característica que ha propiciado un cambio importante en los medios de comunicación: la interactividad. Los medios tradicionales se caracterizaban por una comunicación en un solo sentido; en este momento, el fenómeno de la interactividad es introducido por la innovación tecnológica.

\begin{abstract}
... la imitación de la interacción por parte de un sistema mecánico y electrónico, que contemple como su objetivo principal o colateral también la función de comunicación con un usuario (o entre varios usuarios). Los media interactivos simulan precisa y exclusivamente interacciones comunicativas (...) un diálogo hombre-máquina que haga posible la producción de objetos textuales nuevos, no completamente previsibles a priori (Betettini y Colombo, 1995:17).
\end{abstract}

Los autores señalan que la interactividad tiene tres características:

- Pluridireccionalidad del deslizamiento de las informaciones. Es decir, pueden viajar en múltiples direcciones de manera prácticamente simultánea.

- El papel activo del usuario en la selección de las informaciones requeridas.

- El ritmo particular de la comunicación; la literatura técnica cuantifica esta noción en dos segundos, considerados como tiempo real.

Quizá una de las implicaciones más importantes de la interactividad es la nueva comunicación interpersonal de tipo multimeda, gracias a la cual el usuario puede dialogar con otra $u$ otras personas. Mediante un tipo adecuado de interfa$\mathrm{se}^{5}$ el usuario puede dialogar con otra $\mathrm{u}$ otras personas.
5. Betettini y Colombo entienden por interfase "un dispositivo de tipo informático que permite comunicar dos sistemas que no hablan el mismo lenguaje: por ejemplo, un canal de comunicación que transporta 
datos de tipo analógico y un terminal que procesa datos de tipo digital como puede ser el ordenador" (Betettini y Colombo, 1996:125).Una siguiente acepción considera la estructura de los nuevos medios y admite la comunicación entre dos usuarios. "Se puede decir que todo el sistema que hace posible la comunicación a distancia a través de dispositivos informáticos es una interfase del diálogo entre dos usuarios que "no hablan el mismo lenguaje' en el sentido de que, de otro modo, no estarian en condiciones de comunicarse a causa de su ubicación en lugares distintos $\mathrm{y}$ distantes" (Betettini y Colombo, 1995:125).
La evolución de este tipo de interfase ha hecho que de terminales dotadas con caracteres sólo alfanuméricos (correspondientes a las órdenes enviadas por el usuario y a las respuestas proporcionadas por el sistema o por otros usuarios) se pasase a pantallas en las que aparecen íconos y menús caracterizados gráficamente de modo que resulta posible una memorización y una especificación incluso visual de las posibilidades de acción del usuario y de la estructura de los recorridos que puede realizar" (Betettini y Colombo, 1995:127).

En este sentido, según Betettini y Colombo (1995:36), estos nuevos medios pueden organizarse en torno a sus modalidades productivas en:

- Representación (el uso de un lenguaje adecuado que reproduzca la realidad de la mejor manera posible), como la televisión de alta definición y las posibilidades abiertas por la computadora a partir de la construcción sintética de imágenes;

- Comunicación (modalidades mediante las que se produce el intercambio), nuevamente en el caso de la computadora, la realidad virtual y los equipos multimedia, y

- Conocimiento (posibilidades abiertas para comprender algo o para almacenar y reclamar informaciones $\mathrm{y}$, en general, conocimientos), como el teletexto, el videotel y el CD-ROM, entre otros.

\section{INTERNET}

De acuerdo con la taxonomía de Betettini y Colombo, la computadora y sus interconexiones -internet entre ellasforman parte de los tres rubros considerados representación, porque gradualmente la red de redes ha construido un lenguaje propio, aunque a partir del ya existente en los medios icónicos tradicionales. Para percibir estos cambios graduales basta ver las modificaciones que van sufriendo ciertas páginas en su formato y diseño. Comunicación, por las posibilidades de interactividad abiertas por la red de redes y sobre las que ya se ha adelantado algo. Conocimiento, por las formas crecientes de acceso a información de diverso tipo. De aquí su importancia creciente como medio de comunicación interactivo. 


\section{Conceptualización}

Han pasado más de tres décadas desde que se inició internet, primero como una red destinada a enlazar a los militares norteamericanos para el seguimiento de proyectos estatales y más adelante como enlace entre instituciones académicas para compartir resultados de investigaciones. Incluso, la red se inició en México bajo esta segunda modalidad, aunque en los últimos años se ha desarrollado bajo diversos usos. Dicho brevemente, se trata de una red de computadoras que proporciona acceso a gente e información.

\begin{abstract}
Internet es una suerte de frontera ligeramente anárquica y con mucho espacio no reclamado por explorar (...) Existe una increíble cantidad de información gratuita disponible (...) El futuro también traerá una cantidad importante de información en Internet por la que tendrá que pagar (Hoffman, 1994:17),
\end{abstract}

cosa que actualmente sucede de manera creciente. También prevalece la modalidad de patrocinadores de páginas, de tal forma que la publicidad que circula a través de la red es una de las posibilidades de uso que más ha aumentado en los últimos años.

\section{Usos de internet}

Probablemente el entretenimiento es uno de los usos más importantes de la red de redes. Una revisión de los principales directorios de consulta nacionales e internacionales muestra que una de las tendencias de crecimiento es justamente en este sentido. Incluso, en estos índices de búsqueda los sitios de entretenimiento ya se encuentran ordenados y clasificados para facilitar el acceso del navegante. Por ejemplo, desde hace varios años se han transmitido conciertos de rock vía modem, a la vez que el cine y la televisión han encontrado en la red un espacio para la reproducción y complemento de sus materiales ${ }^{6}$.

En este sentido, el creciente interés por el entretenimiento justifica, en buena medida, el considerable aumento en el número de páginas dedicadas a la temática del fútbol, particularmente desde los aficionados que pretenden establecer comunicación tanto con su equipo como con simpatizantes localizados dentro y fuera de su país. Sin embargo, como se verá más adelante, no debe perderse de vista que tras buena parte de estas páginas también subyace un
6. Para mayor información al respecto véase Trejo, 1996. 
interés informativo. Esto es evidente en las páginas oficiales así como en las pertenecientes a las asociaciones nacionales e internacionales, aunque también la hinchada busca ir más allá del entretenimiento y trascender en su página. Esto conduce a una discusión que desde hace varios años ha prevalecido para la televisión: la posibilidad de hablar de entretenimiento puro, de entretenimiento como información -el caso de los programas de concurso-, de información como entretenimiento -del deporte- y de información como información, que sería el caso de los noticiarios (Baggaley y Duck, 1980). Volveremos a este punto al exponer los resultados de la encuesta.

Por otra parte, si bien el entretenimiento es uno de los usos más importantes de la red de redes, por el tema que nos ocupa cabe también señalar los siguientes:

- Correo electrónico.- Por su instantaneidad y bajo costo justifica la incorporación de las empresas a internet. Estas características han modificado las formas de acceder e intercambiar información tanto entre comunidades reducidas como entre crecientes grupos de interés. En pocas palabras, se trata de un sistema electrónico de información directa. Es fácil de contestar, aun cuando los interlocutores no se conozcan, a través de la dirección. En principio, el correo electrónico se diseñó para permitir la comunicación entre dos personas por medio de computadoras. Actualmente puede utilizarse con otras posibilidades (Comer, 1995:143), pues un mismo mensaje puede enviarse a varias personas, además de que actualmente es posible que este mensaje incluya texto, voz, video o gráficos.

- Comunidades de discusión.- Conocidos como grupos de discusión, foros, clubes, grupos de noticias o tableros electrónicos de boletines. Para fines de este trabajo cabe destacar los siguientes:

- Listas de distribución de correo, grupo de correo o grupos de suscriptores. Consiste en un listado de personas interesadas en un tema específico, que desean recibir información al respecto. En el caso del fútbol es frecuente encontrarlas al menos bajo dos modalidades: un determinado equipo o jugador que suscita diversos puntos a discutir, o una temática en particular sobre la que se invita a discutir. Para participar uno se suscribe en una o varias listas y recibe la información vía correo electrónico. 
- Servicio de boletín electrónico o noticias en red. Permite al usuario participar en uno o varios grupos de discusión y en las discusiones con los miembros del grupo. Abre posibilidades a la participación en diversos grupos de discusión, cada uno enfocado a un tema específico. De acuerdo con Douglas Comer (1995:197-198) el boletín electrónico cumple la misma función que el tablero de boletines en la vida diaria: colocar noticias que otros puedan leer. Normalmente las posibilidades de participación del interesado se dan desde una determinada página, desde donde revisa si han aparecido nuevos materiales y envía notas tanto al grupo de discusión para que todos la lean o como respuesta a lo que alguien haya escrito sobre algún tema. En el tema que nos ocupa, esta participación se hace desde la página elegida por el usuario.

- Artículos de Usernet. Constituyen una de las mejores maneras de mantenerse informado sobre algún tema en particular y uno de los pilares de internet. Permiten un mejor intercambio de conversaciones. Todos los comentarios de una persona se envían a todos los integrantes del grupo de interés. Los usuarios pueden responderlos o no, dependiendo de los intereses. En fútbol encontramos que también es frecuente recurrir a esta modalidad, de manera similar a la lista de distribución de correo.

Para concluir con este apartado, cabe insistir en que los usos de internet descritos para efectos de este trabajo constituyen las modalidades señaladas por Betettini y Colombo por su aportación a la comunicación interpersonal de tipo multimedia. Ciertamente, estas posibilidades incrementan el espectro de relaciones del individuo, sobre todo por el contacto que puede establecerse con personas afines ubicadas en distancias alejadas. Es decir, se trata de una de las características de la sociedad digitalizada: la eliminación de fronteras geográficas, sumada a la posibilidad de unir a personas que se encuentran ubicadas en lugares distantes.

\section{Fútbol e internet}

Al buscar soccer, calcio o fútbol en cualquiera de los índices se corre el riesgo de enfrentarse a cerca de 20 mil posi- 
bilidades por índice. Los navegantes más especializados buscarán la información bajo el nombre de su equipo, con lo que se corre un nuevo riesgo: que bajo el nombre que uno busca se encuentran otras referencias similares. Por ejemplo, al genérico Alianza corresponden 49,330 referencias en Alta Vista; 219 en Web Crawler, nueve en Yahoo -entre los que se encuentra uno de los equipos favoritos de la hinchada peruana-, para frustración de los partidarios del Alianza de Chile, e infinitas posibilidades en Lycos, para citar algunos ejemplos. Obviamente Yahoo será la opción más rápida para anotar el gol; algo que no siempre saben los usuarios, sobre todo en sus primeras experiencias en la red. Por tanto, acceder a los directorios de búsqueda supone una mayor precisión, la que se va adquiriendo mientras más experto se es en el tema y en la navegación a través de internet.

La otra posibilidad de acceder a información sobre fútbol es a través de páginas especializadas y tres son las más importantes para los fines de este trabajo, por tratarse de espacios específicos en internet dedicados al fútbol: ISFA (siglas de Internet Soccer Fans Association), la Unión de Clubes Latinoamericanos en Internet y Soccer City. Entre otros servicios ISFA ofrece a los tifosi la oportunidad de contar con su propia página de fútbol o ubicar la que ya tienen en esta dirección. En principio, ambas oportunidades permitirán que, una vez ubicadas en una página especializada, habrá un mayor número de visitantes. Un siguiente servicio, que no se contrapone a los otros, es la participación de grupos de discusión sobre diversos temas; para ello cuenta con 433 listas de correo. Los interesados en participar en estos grupos pueden seleccionar a sus equipos favoritos por continente $\mathrm{u}$ otras listas, en las que la participación se registra por juegos, novedades y jugadores, entre otras modalidades.

Asimismo, los interesados pueden añadir nuevas listas sobre temáticas de interés, las mismas que prácticamente de inmediato quedan anotadas en ISFA. En cuanto a los equipos, uno puede acceder por orden alfabético a páginas de equipos en particular o de algún país. Cabe destacar que mientras el fútbol europeo tiene una presencia importante en estas páginas -todas de aficionados-, únicamente Brasil, Uruguay, Colombia, Chile y Perú están presentes en ISFA, aunque el primero con un número más elevado de equipos. En cuanto a los chat rooms, el usuario puede conectarse mediante correo electrónico con el grupo o tema de su preferencia. En este caso, la mayoría de los tópicos se relaciona con equipos de fútbol. Volveremos a este punto más adelante. 
Por su parte, la Unión de Clubes Latinoamericanos en internet es todavía una organización incipiente que cuenta con páginas en la red de redes. En principio únicamente cuentan con un listado de 15 páginas elaboradas por aficionados de igual número de clubes. Hay un lazo a cada una de estas páginas y a la dirección electrónica de sus autores. Asimismo, cuenta con acceso a siete índices de búsqueda a través de los que puede obtenerse información no sólo sobre fútbol, sino sobre cualquier otro tema. Finalmente, Soccer City reúne un número más elevado de páginas, tanto oficiales como de aficionados, agrupadas también por continente, país y equipo. Ofrece también servicios especiales para los $t i-$ fosi, en lo que se refiere a formas de obtener una página propia y de acceder a grupos de discusión.

Finalmente, hay otras modalidades de acceder al fútbol mediante internet, particularmente a través de las asociaciones nacionales -como la Federación Mexicana de Fútbol y sus homólogas en cada país-, regionales como la Concacaf, Conmebol o internacionales como la FIFA. Asimismo, en casos como Argentina, Chile, Perú, España e Italia hay páginas que concentran -en páginas nacionales- a un buen número de equipos que cuentan con uno o varios de los servicios enlistados en el apartado correspondiente a los usos de internet.

\section{EL FÚTBOL EN EL CIBERESPACIO}

\section{Los inicios}

En 1995 comienza a popularizarse la red de redes entre sus usuarios potenciales. En este mismo año se inicia el furor de las páginas en internet. Algunos hinchas comenzaron a elaborar páginas sobre sus equipos favoritos. Francisco José Membrilla, del Atlético de Madrid y Jordi Praderas, del Espanyol, iniciaron por separado sus páginas para que sus equipos tuvieran presencia en el ciberespacio, pues entonces no la tenían. Lo mismo sucedió con los catedráticos de la Facultad de Informática de la Universidad de Sevilla, quienes crearon las páginas del Real Betis Balompié cuando había muy poca información sobre el tema, además de que actualizaban todas las plantillas de los equipos de primera división del fútbol español. Los tres casos se consideran pioneros de lo que vendría más adelante, tanto para el fútbol español como de todo el mundo. Otros aficionados -y en algunos casos la directiva oficial- rápidamente poblaron el ciberespacio con 
otras páginas, sobre todo de sus aficionados que pronto mostraron sus potencialidades de comunicación con los afines e incluso con los enemigos.

Por otra parte, una primera visita realizada a más de cien páginas de internet muestra que, al menos a primera vista, la mayoría de las páginas dedicadas a un solo club, sea cual fuere, contienen la misma información aunque diseñada y desarrollada de diversas maneras, en función de intereses y conocimientos particulares. Todas contienen información de su club, resultados de la jornada, materiales adicionales que varian de un lugar a otro -como grupos de discusión o tableros de anuncios- y un espacio para comentarios de los visitantes. Esta primera observación remite a una pregunta formulada por estudiosos de los medios de comunicación, tradicionales o nuevos, referida a la validez de manejar la misma información aunque de manera diferente. La consecuencia de esta primera observación y esta pregunta dio origen al resto de esta investigación.

\section{La encuesta}

Para dar una idea del crecimiento del fútbol en el ciberespacio, se aplicó una encuesta a 100 usuarios de páginas de internet relacionadas con el fútbol, de los cuales respondieron 91. Dicha encuesta tuvo un carácter exploratorio, en la medida en que buscó "tener un acercamiento al fenómeno estudiado (así como) para identificar las características generales o dimensiones del problema, así como para establecer hipótesis y alternativas de trabajo" (López Romo, 1998:38). La tabla resume la procedencia de la información; de aquí en adelante la información se manejará en porcentajes, para mostrar tendencias con respecto al uso de internet por la hinchada futbolera: 


\begin{tabular}{lrr} 
Procedencia & Num. & \% \\
\hline Argentina & 18 & 19,87 \\
España & 15 & 16,48 \\
Italia & 14 & 15,38 \\
México & 10 & 10,98 \\
Perú & 7 & 7,69 \\
Francia & 6 & 6,59 \\
Chile & 5 & 5,49 \\
Brasil & 5 & 5,49 \\
Uruguay & 4 & 4,39 \\
Estados Unidos & 2 & 2,19 \\
Suecia & 1 & 1,09 \\
Ecuador & 1 & 1,09 \\
Inglaterra & 1 & 1,09 \\
Guatemala & 1 & 1,09 \\
El Salvador & 1 & 1,09 \\
Total & $\mathbf{9 1}$ & $\mathbf{1 0 0 , 0 0}$ \\
\hline
\end{tabular}

Como puede observarse, la tabla resume el número de páginas por país. En prácticamente todos los casos la presencia de los aficionados es mayor que la de los directivos. Además, hay una gran dispersión, puesto que son muy pocos los equipos que cuentan con más de una página, como puede ser el Atlético Industrial de Argentina, el Atlético de Madrid, el Zaragoza y el Betis de España, el Torino de Italia o el Alianza Lima de Perú. Más bien la constante suele ser la dispersión. Por ejemplo, en Argentina, 16 páginas corresponden a hinchas del Lanús, Racing, Quilmes, Cañuelas, Rojo, Atlético Unión, Ferrocarril Oeste, Avellaneda, Rosario Central, San Martín de Tucumán y Tigre, además del ya mencionado Atlético Industrial. La única página oficial corresponde al Atlético Independiente y la última pertenece a un programa de televisión que tiene un espacio deportivo en internet.

En España la situación es similar, pues 14 aficionados tienen su página y una es oficial: la de Orgullo Vikingo. Además de los tres equipos mencionados, el Barcelona, el Real Madrid y el Alavés han merecido la atención de su público. También encontramos cinco páginas que no pertenecen a un club en particular; más bien se trata de grupos de aficionados que se organizan para crear sus páginas de fútbol (Alabarces: el fútbol supone irle a un club). El caso de Italia difiere del resto. Además del Torino, el Milan, Piacenza, Juventus y Genoa cuentan con espacios por cortesía de sus hinchas. El Lecce, el Lazio y el Sampdoria tienen páginas oficiales. Merece especial 
atención este último caso, pues cuentan con tres páginas que mantienen y apoyan como una forma de establecer contacto con la afición proveniente de diversas partes del mundo.

Por su parte, la situación se acentúa en Francia, en donde el Guincamp, el Cannes y dos aficionados del Auxerre cuentan con sus páginas, mientras que en Chile el Antofagasta, la Universidad Católica, el Colo Colo y el Coquimbo están presentes en la red vía sus tifosi. Lo mismo sucede con el América de Río, el Cruzeiro Sporte Club de Brasil o el Nacional y el Peñarol de Uruguay.

Por otra parte, en la mayoría de los casos la procedencia de la página coincide con el país de origen. Sin embargo, en 1995, cuando Luis llegó a Estados Unidos procedente de Argentina y empezó a navegar en internet, se encontró con muy poca información sobre los equipos de su país. Por ello se propuso hacer una página que contuviera información sobre el tema, que sirviera de referente para hinchas que, como él, viven fuera de su país. Esta razón motivó también a Jaime, quien llegó a Miami procedente de Perú, y desde ahí cuenta con una página de internet dedicada especialmente al Alianza Lima. Asimismo, en abril de 1995 Marinho viajó de Brasil a la Universidad de Newcastle a continuar con sus estudios de posgrado. Ahí comenzó a extrañar la información sobre Gremio, su equipo favorito, por lo que hizo su página para compartir con los interesados en el fútbol brasileño.

En suma, la procedencia de la información muestra que al menos existen tres tipos de páginas: las oficiales -un $14,28 \%$ del total de la muestra-, entre las que llaman la atención los equipos italianos Lecce y Sampdoria con tres y dos páginas oficiales respectivamente. Por su parte, la Federación Guatemalteca de Fútbol cuenta con su página para informar cotidianamente a nacionales y extranjeros sobre la situación del fútbol en su país. Por su parte, un 6,59\% de las páginas corresponden a los medios de comunicación locales de distintos países, que cuentan con páginas en internet por diversos motivos. Por ejemplo, Depornet en Ecuador se considera el primer grupo en Sudamérica que aprovechó la red para el manejo del fútbol; son profesionales que, a propósito del fútbol, acercan a ecuatorianos dentro y fuera del país, a la vez que proporcionan servicios informativos a estaciones de radio y televisión. En Argentina y El Salvador hay dos páginas de internet que son extensiones de programas de televisión cuyo eje temático es el fútbol. Finalmente, el 79,13\% restante corresponde a la hinchada que, como ya se indicó y se ampliará en el siguiente apartado, cuenta con su página por diversas razones. 


\section{Sobre las razones para tener una página en internet}

Quienes mantienen las páginas oficiales destacan la necesidad de entrar en contacto con la afición dentro y fuera del país de origen. La muestra que integra este estudio destaca que Italia es el país en el que hay una mayor conciencia al respecto; a través de sus tres páginas entran en contacto con los tifosi de todo el mundo. Como ya se destacó, el Lecce y el Sampdoria ocupan lugares importantes en este sentido. Por otra parte, el Atlético Independiente de Argentina, el Orgullo Vikingo de España, los clubes Cruz Azul, Chivas y Tecos, ambos de Guadalajara, en México, el Corinthians, en Brasil y el Peñarol en Uruguay cuentan con su página por las mismas razones.

A pesar de que el principal motivo que aducen los clubes que mantienen páginas oficiales es el contacto con el público, lo cierto es que la página en internet tiene otros usos vinculados a la industria del fútbol: desde la facilidad de acceso vía correo electrónico, lo que permite el contacto inmediato con clubes y asociaciones nacionales e internacionales, hasta los convenios con firmas publicitarias que crecientemente se dedican a producir y subir información de este tipo a través de la red de redes. Entonces, a la comunicación interpersonal cabe añadir las finalidades publicitaria y económica; ambas van de la mano, aunque se separan en ciertos momentos.

Por su parte, los aficionados señalan un conjunto de razones que pueden sintetizarse de la siguiente manera:

- El hincha individual es el más entusiasta participante del ciberespacio. Aunado al auge de la computación que de manera creciente se enseña desde la primaria y la secundaria en escuelas públicas y privadas, hinchas del Real Madrid, del Atlético de Madrid y del Deportivo Alavés en España; del Alianza Lima, de Perú; del Racing Club de Avellaneda, en Argentina, y de los Tigres de la Universidad de Nuevo León, en México, decidieron poner sus conocimientos al servicio de sus dos pasiones más importantes: el fútbol y la computación.

- De la misma forma, grupos de hinchas del Atlético Unión y del Lanús de Argentina; del Veracruz en México, entre otros, se unieron para hacer la página de su equipo, la misma que mantienen y actualizan en grupo. Por ejemplo, Carlos Jaureche y sus amigos realizan este trabajo desde Uruguay, a juzgar por ellos, con muy buenos resultados. 
- También hay infinidad de hinchas individuales que provienen de diversas partes y que tienen su página bajo diversas modalidades: independientemente de su Club, como el Atlas, de México o el Racing y el Atlético Unión de Argentina; el hincha de este último enfatiza incluso que no debe cobrarse al club por este servicio, pues recibir dinero es traicionar a la hinchada. Otra modalidad de participación en el ciberespacio es que los hinchas lleguen a ligarse a los directivos de su equipo como el Alianza Lima, el Nacional de Uruguay o el Cruz Azul de México, que pronto se convirtió en la página oficial del club.

En todos los casos hay una importante motivación que orienta su trabajo: que la hinchada de todo el mundo sepa que hay otros equipos de fútbol y no los que ya conoce por ser los más famosos. En menor medida, a través de su trabajo los tifosi pretenden entrar en contacto con su respectivo club, ya sea en busca de información o de apoyos de otro tipo. En algunos casos la respuesta ha sido favorable, pues quienes empezaron trabajando de manera independiente ahora lo hacen apoyados por su club, como Alex, hincha del club Universitario de Deportes, del Perú o Francisco Cabeza, aficionado del mexicano Cruz Azul. En otros casos, la respuesta no ha sido tan favorable pues el club opta por su propia página o simplemente por no hacer caso a los tifosi.

\section{Sobre la actualización de la información}

En las páginas oficiales y en las que participan periodistas, tienen mayores posibilidades de mantenerse al día, aunque ambas por diferentes razones. Las primeras, porque hay un compromiso tanto con la afición como con los medios, clubes y organizaciones locales, regionales e internacionales que demandan esta información. Los medios y agencias, porque en la mayoría de los casos han establecido contratos con medios locales y regionales -en algunas ocasiones internacionales- que igualmente demandan información actualizada que permita alimentar sus servicios informativos. Incluso, estas instancias cuentan con periodistas especializados que se encargan de este trabajo, como ocurre en Guatemala y Ecuador, ya descritos. Asimismo el Corinthians, de Brasil, cuenta con un periodista contratado para mantener la página.

Por su parte, los tifosi que tienen sus páginas por afición o por informar a quienes están dentro y fuera del país 
revisten más problemas de actualización. Muchos son estudiantes que dan prioridad a sus actividades, las cuales dificultan su trabajo, sobre todo en período de exámenes. Sin embargo, en mayor o menor medida todos reconocen la importancia de actualizarlas y de mantener en sus páginas archivos de consulta sobre información del tiempo pasado. Nuevamente llama la atención el caso de los colegas de la Universidad de Sevilla, quienes han pensado en borrar sus páginas, aunque no lo hacen por los lazos que mantienen con equipos e hinchas que les han pedido ser incluidos en ese espacio. Pero la constante es la actualización una vez por semana al menos, después de los resultados de la jornada dominical. Otros piensan en hacerlo tan a menudo como sea necesario, pero en general depende de la composición de sus páginas. La actualización está más ligada al tiempo libre y, por tanto, al entretenimiento que a las necesidades propias de la información.

\section{Sobre la semejanza de la información}

Como ya se indicó en el apartado anterior, uno de los aspectos que dio origen a esta investigación fue justamente el carácter repetitivo de parte de la información incluida en la mayoría de las páginas. De los 91 encuestados 13 no hicieron comentarios al respecto, muchos de ellos porque señalan no haber entendido el sentido de la pregunta. Cinco no revisan mientras que 73 tienen opiniones diferentes que proporcionan un panorama sobre las concepciones y usos de la red de redes:

- $\quad$ El 23,07\% de quienes respondieron la encuesta señalan o bien no comprender la pregunta o no estar interesados en si hay páginas similares a la suya. Quizá esta respuesta tendría menos implicaciones si viniera de hinchas que consideran tener la mejor información y que no necesitan ver lo que hace el resto de la hinchada o las páginas oficiales de su club. Sin embargo, en varios casos de páginas oficiales se encuentra esta respuesta e incluso en páginas manejadas o administradas por medios de comunicación. El problema cobra aquí otras dimensiones que quizá aluden al profesionalismo con que se hace un trabajo, al no conocer lo que hace la competencia. En el mejor de los casos, quienes manejan, administran o actualizan estas páginas se orientan por los comentarios que reciben vía correo 
electrónico, sobre lo que se hablará más adelante. Cabe adelantar que estos comentarios proporcionan únicamente un punto de vista, el del usuario que responde. Habría que ver si este punto de vista da cuenta de las necesidades informativas de los usuarios de la página.

- Un 5,49\% reconoce que efectivamente se trata de la misma información. El consenso es que si únicamente hubiera una página de tal o cual equipo, la información sería mejor y ayudaría a que no se repitiera. Incluso, hay quienes han pensado en borrar sus páginas ante la misma redundancia que resta sentido a su trabajo, como es el caso de los colegas de la Universidad de Sevilla.

- $\quad$ El 71,44\% corresponde en su mayoría a hinchas y un pequeño porcentaje a administradores de páginas oficiales y a servicios informativos o medios de comunicación. Llama la atención el hecho de que esta mayoría tiene percepciones que van muy de acuerdo con los principios bajo los cuales funciona la red de redes:

- Por una parte, se considera que la posibilidad de que haya una abundante circulación de información es una característica de la red de redes. Bajo esta idea subyace la posibilidad de que el hincha acceda a la información localizable tanto mediante el azar -con los riesgos ya descritos- como mediante direcciones que concentran información especializada, en este caso sobre fútbol.

- Otro porcentaje considera que la redundancia informativa, que también puede ser característica de internet, obliga a quien tiene la página a ser mejor que el resto. En consecuencia, es bueno tener competencia de dónde elegir; a final de cuentas, cada quién diseña su página a su manera y el aficionado buscará las alternativas que satisfagan sus necesidades. En suma, deben predominar la calidad y el buen servicio.

- Otro enfoque que se desprende del anterior considera que cada administrador de página le imprime su propio sello a la información que sube a internet. De aquí que en el diseño personal de las páginas radique la posibilidad de que los 
hinchas se expresen libremente a través de la red, respetando las leyes del hipertexto -como sucede en la mayoría de los casos- y actualizando no sólo la información sobre el tema sino también el diseño de la página.

- Derivado de lo anterior hay quienes destacan la importancia de estar en la red y sus potencialidades comunicativas para el futuro. En este sentido, se argumentan la interactividad de la red y la posibilidad de acceder a información procedente de diversas partes del mundo.

- Otro tipo de comentarios aduce las responsabilidades éticas que deben caracterizar a quienes cuentan con su página en la red de redes: la presentación de información verdadera, que el usuario pueda manejar con confianza. Incluso, en este punto de vista se argumenta la posibilidad de que el usuario visite varias páginas y que se forme una opinión personal sobre la temática de su interés.

- Hay quienes destacan que son la única página de su club. Esto los convierte en especialistas en un tema que no todos manejan. En consecuencia, los hinchas accederán a sus páginas sabiendo de antemano que se encuentran con información novedosa, dificilmente localizable en otras páginas.

- Finalmente se alude a la piratería. Es decir, a la posibilidad de que la información novedosa y actual se convierta a su vez en fuente de información para otras páginas. Algo que, también argumentan, resulta dificil de comprobar. En este sentido, llama la atención que los hinchas que están en esta situación señalan haberla comprobado y la asumen positivamente como uno de los riesgos de internet.

\section{Sobre los comentarios recibidos}

Los 91 cuestionarios contestados señalan haber recibido comentarios a sus páginas bajo las siguientes modalidades:

- En muchos casos, particularmente el Milan, el Real Madrid y el Alianza Lima, el club favorito trasciende las fronteras geográficas. Recibir comentarios pro- 
cedentes de puntos distantes de donde se ubica el club son ilustrativos, pues unifican a la hinchada y muestran la fortaleza adquirida por el club. Esta afirmación puede hacerse extensiva a las páginas oficiales, cuyos administradores se muestran sorprendidos de la cantidad de tifosi que tiene su club, cuya procedencia es local o de otros países.

- Quienes cuentan con sus páginas en el ciberespacio también se muestran sorprendidos ante este nuevo medio de comunicación, pues los visitantes de sus páginas les proporcionan información que puede serles de utilidad tanto para mejorar sus páginas -en términos de formato- como datos y cifras que pueden incluir.

- En mayor o menor medida, todos reconocen que reciben mayor cantidad de mensajes durante la temporada, pues es el momento en que la hinchada hace sus comentarios con respecto al desempeño de su equipo y de los adversarios, la forma en que se comportan los asistentes al estadio y los medios de comunicación, así como las opiniones de la directiva y de la propia hinchada al respecto.

- La mayoría señala recibir una buena cantidad de felicitaciones por las páginas. En los casos pertinentes, estas felicitaciones se acompañan de reclamos por no actualizar la información tan seguido como es necesario.

- Se reciben mensajes publicables en los tableros de anuncios o que más adelante formarán parte de los grupos de discusión.

- Finalmente, miembros de equipos contrincantes también acceden a las páginas rivales y hacen comentarios adversos, tanto sobre la página como sobre el desempeño del equipo, anticipando su derrota en el momento crucial de la temporada.

En suma, la gran circulación de comentarios a través del ciberespacio pone de manifiesto que, sin lugar a dudas, internet se ha erigido en un ámbito que propicia la comunicación interpersonal mediada por la computadora. No obstante, no podemos pecar de optimistas pues se trata de una 
modalidad de interactividad y no de interacción, la que únicamente puede llevarse a cabo cara a cara.

De cualquier manera, es evidente que los ciberhinchas han encontrado en la red de redes un importante espacio de expresión tanto de sus intereses como de un lenguaje propio.

\section{CONCLUSIONES}

Hasta aquí la presentación de los resultados preliminares de la encuesta aplicada a 91 ciber-tifosi. Cabe aclarar que estos resultados son representativos de un cierto número de usuarios, aunque todavía queda mucho por explorar al respecto.

Por ejemplo, es claro que hay muchas más páginas oficiales que las que respondieron la encuesta, así como hay más páginas de servicios informativos y de ciberaficionados. Por lo tanto, esta encuesta debe tomarse como un primer acercamiento a las modalidades como el fútbol se acerca al ciberespacio.

Asimismo, en función de la manera en que se ha presentado la información, las conclusiones se presentarán bajo dos modalidades: las posibilidades de acceder a la gramática del hipertexto y el nuevo espacio apropiable por la ciberhinchada.

\section{Sobre las posibilidades de acceder a la gramática de hipertexto}

Por sus características, la encuesta no incluyó la edad del ciberaficionado. Sin embargo, puede inferirse que a internet ingresan usuarios de dos generaciones: los adultos que por razones profesionales han tenido que acceder a la red de redes, aunque apoyados por un equipo técnico que les facilita este trabajo, y los jóvenes que han tomado uno o varios cursos de computación como parte de su formación escolar y que ponen a prueba sus conocimientos con la página de su equipo favorito. Ya en su momento se habló del importante número de ciber-tifosi que a través de sus páginas combinan sus dos pasiones: la computación y el fútbol. Sin temor a equivocarnos, podemos decir que ambos grupos irán en aumento, aunque el segundo sin comparación al primero, dadas las proyecciones que están adquiriendo estos contenidos en la educación; por el momento son pocas las instituciones que pueden y asumen la enseñanza de hipertexto y de diseño de páginas en internet. Ciertamente este número va en aumento. 
Sin embargo, y nuevamente por las limitaciones del estudio, es difícil detectar quiénes están preparados para tener su página en tanto conozcan la gramática y las leyes del hipertexto. Es decir, a primera vista uno percibe que hay páginas mejor diseñadas que otras, o formatos más modernos que otros; mientras tanto, todavía son pocos los materiales que dan cuenta de la teoría del hipertexto y de cómo respetar sus leyes (por ejemplo Landow, 1995; Burdea, 1996). En la medida en que se conozcan la gramática y las leyes del hipertexto se accederá a páginas con mayor calidad y con una mejor definición, que además aseguren una adecuada colocación de la información y el buen servicio al usuario que quiere dar buena parte de los ciberhinchas entrevistados para este trabajo. Después de todo, una buena información debe acompañarse de un buen formato. En este mismo sentido, en la medida en que los ciberviajeros adquieran una mayor competencia hipertextual habrá una mayor demanda de calidad del servicio, en términos de información y de formato.

Asimismo, como lo mostraron los datos analizados, existe conciencia por parte de los ciberhinchas sobre la necesidad de actualizar las páginas en internet. Incluso algunos lo señalan como parte de un reclamo de sus visitantes. Esta inquietud muestra que hay una primera competencia con respecto a los usos y necesidades de internet: la página que no es periódicamente actualizada va perdiendo visitantes por muy interesante que sea la información con que se le alimentó la primera vez. Sin embargo, particularmente en los hinchas esta inquietud no está presente en la medida en que, a pesar de que se reconoce la necesidad de la actualización, ésta se posterga para los tiempos libres. Por lo tanto, si bien se reconoce la importancia de la información, a la que contiene la página se le coloca como parte del entretenimiento. Una especie de contrasentido que prevalece en buena parte de las páginas de los ciber-tifosi y que, como ya se indicó, no está presente en las páginas oficiales o las de servicios informativos, en las cuales la información es considerada como información (tal como lo muestra el énfasis en las noticias y en las estadísticas) o como entretenimiento, al abrir espacios de contacto para con la ciberafición y entre ellos mismos.

\section{Apertura de un nuevo espacio para el deporte}

Críticos y defensores de la red de redes recurren al mismo argumento como centro de su discurso: el hecho de que 
internet elimine las fronteras geográficas propicia la creación de un ámbito ilusorio en el que se eliminan las diferencias económicas, políticas y socioculturales de los usuarios, tanto dentro de sus países como hacia afuera. Esta encuesta es una constatación más. Después de todo, se dispone de muy poca información que muestre la pertenencia a una clase social o el nivel socioeconómico de los usuarios. Hay quienes respondieron desde la dirección electrónica ubicada en su casa, en su centro de trabajo o en la institución en la que estudian. El hecho es que en nuestros países la presencia de internet agudiza la discusión sobre este tipo de fenómenos que, por sus características y dimensiones, trascienden los limites de este estudio.

Por otra parte, los resultados de la encuesta y los comentarios al respecto ponen de manifiesto que la red de redes se ha constituido en un importante espacio de expresión de la ciberhinchada procedente de diversas partes del mundo. A la vez que les permite dar a conocer su equipo favorito bajo diversas modalidades, hoy más que nunca tienen un importante acceso a comentarios procedentes del extranjero, tanto de nacionales que han tenido que salir de su país como extranjeros que miran con sorpresa que uno de sus equipos favoritos tiene un espacio en la red. Como ya se indicó, esto ha propiciado nuevas modalidades de comunicación interactiva que asumen las características de comunicación interpersonal. Es decir, se le evalúa con un excesivo optimismo, pues a final de cuentas habria que preguntarse si hay algún compromiso adicional más allá del adquirido por ser ciberhinchas del mismo equipo.

Así, evaluar esta nueva modalidad comunicativa supone ubicar el fútbol en el contexto de la globalización misma que, tarde o temprano, conducirá a modificaciones importantes en el trabajo, las aficiones y la propia comunicación. Algo sobre lo que también será necesario estar alertas, pues es probable que apenas estemos viendo los primeros visos de lo que se avecina. Prueba de ello es la manera en que los ciber-tifosi asumen la piratería de sus materiales -aunque ninguno reconoce haberlo hecho- como forma de acceder a información y como una consecuencia de acceder a la red de redes. En otro sentido, el acceso a información especializada sobre el club del ciberaficionado constituye una muestra de cómo circula la información en la era digital, la misma que incluye datos sobre la persona que determinan el tipo de servicios a recibir en comunidades cada vez más reducidas.

Finalmente, no puede perderse de vista que en el proceso de globalización del fútbol han tenido mucha importancia 
7. Para el caso de México, cuando Hugo Sánchez -para una inmensa mayoría el mejor jugador en mucho tiempo- comenzó a jugar primero en el Atlético de Madrid y después en el Real Madrid, el número de aficionados mexicanos de estos equipos se elevó considerablemente. De ello fueron conscientes las televisoras, particularmente Televisa, la misma que hizo los arreglos necesarios para la cobertura de los juegos de este equipo en las diversas temporadas en que jugó Hugo

Sánchez. los medios tradicionales de comunicación, particularmente la televisión. Es a través de este medio que de las transmisiones locales, regionales y más adelante nacionales, se pasó a la transmisión de partidos de fuerte arraigo local y nacional -aunque con jugadores y directores técnicos procedentes de diversas partes del mundo-, lo cual ha garantizado la consolidación e internacionalización de jugadores, directores técnicos y equipos ${ }^{7}$. En este proceso ha participado activamente una característica de la televisión todavía no igualada por otro medio de comunicación tradicional y nuevo: la simultaneidad.

Así, habrá partidos -como los del campeonato mundialcuya audiencia potencial se estima en los 2.000 millones de habitantes ubicados en todo el globo. Salvo esta excepción, el resto contará con una audiencia potencial menor, únicamente estimable por el número de tifosi que se tenga en diversas partes del mundo. Un dato que depende de infinidad de variables: desde la nacionalidad de jugadores y director técnico hasta los intereses manifestados abierta o veladamente por la hinchada y que explican el fenómeno del Boca Juniors o del Inter de Milán. Todavía internet presenta dificultadas para captar simultáneamente a un número importante de tifosi. Sin embargo, previsiblemente puede constituirse en un importante medio de expresión y comunicación. Habrá que ver si para ello se respetan no sólo la gramática y las leyes del hipertexto, sino las del fair play, las de la transmisión de información veraz por canales auténticos y no por la piratería. 
Bibliografía

Baggaley y Duck

1980

Análisis del mensaje televisivo.

Barcelona: Gustavo Gili.

Bañuelos Rentería, Javier

1998

Balón a tierra (1896-1932), primer tomo de la Crónica del fútbol mexicano. México DF: Clío.

Benassini, Claudia 1997

Internet como apoyo a la investigación de televisión. Cuadernos de Mass Culturas. México DF: Universidad Iberoamericana Plantel León.

Betettini, Gianfranco y Fausto Colombo

1995

Las nuevas tecnologías de la comunicación. Barcelona: Paidós.

Burdea, Grigore y Philippe Coiffet

1996

Tecnologías de la realidad virtual. Barcelona: Paidós.

Comer, Douglas E. 1995

El libro de internet. México DF: Prentice Hall.

Giddens, Anthony 1994

Consecuencias de la modernidad. Madrid: Alianza Editorial.

Hoffman, Paul 1994

Internet, manual de bolsillo. México DF: McGraw-Hill.

Landow, George 1995

Hipertexto: la convergencia de la teoría crítica contemporánea y la tecnología. Barcelona: Paidós.

López Romo, Heriberto 1998

"La metodología de la encuesta" en Galindo, Jesús et.al. Técnicas de 
Negroponte, Nicholas

1996

Trejo, Raúl

1996 investigación en sociedad, cultura y comunicación. México DF: AddisonWesley-Longman.

Ser digital. México DF: Océano.

La nueva alfombra mágica. México DF: Diana. 